\title{
Research and development of automatic gear shifting system for automobile manual transmission
}

\author{
Cheng Tianyou, a , Liang Kuankuan ${ }^{1, b}$, Yang Xiong ${ }^{1, \mathrm{c}}$, Gong Yunpeng ${ }^{1, \mathrm{~d}}$ \\ ${ }^{1}$ School of Northeastern University, Shenyang 510000, China;
}

a68231775@qq.com ,b1187263290@qq.com,c1573353955@qq.com, dypgong@mail.neu.edu.cn

Keywords: Life testing bench, Manual transmission box, Automatic transmission, Multi-position cylinder, control;

\begin{abstract}
Controlling speed mechanism of The National 863 Program "typical product operation safety life prediction technology research" test bed is the automobile manual test bed. In order to achieve shifting gears automatically for that test bed, this article introduces one system which uses screw and cylinder to drive the shifting rod to move in two different directions. The experimental results show that the system can be more stable than human shift, it can drive the shifting rod regularly and circularly.
\end{abstract}

\section{Introduction}

The automotive transmission can be roughly divided into three categories, one is manual transmission (MT); one is the automatic transmission; the third is a manual automatic integrated transmission[1].Most of the current domestic heavy truck transmission is automatic shift structure[2],the transmission has the essential importance for the vehicle's power and economy,the reliability and portability of shifting and the stability efficiency of transmission[3].Automatic mechanical transmission (AMT) has the advantages of high transmission efficiency, compact structure and high reliability [4]. At present, in the domestic and foreign manual transmission testing equipment, the person as the driver come to choose file and shift, because each operator manipulation habits are not the same, for the shift of the force, displacement control is different, that directly affected the shifting quality objective evaluation, for manual transmission shift experiment, critical part of Technology is how to achieve automatic shift for the manual transmission[5]. The current European has about 400 thousand vehicles equipped with automatic transmissions, the number of cars using automatic transmission is increasing sharply[6].

Differed in other automobile manual transmissions, this system is aimed to replace the simple repetition of manual shifting action, achieving the accurate and efficient automatically shifting action, to improve working efficiency and shifting quality, so it can avoid experimenters make the misoperation due to fatigue and other factors, provide reliable speed switch security for the life test station. According to the requirement of national and industry of automotive manual transmissions of test, the paper puts forward using screw and multi-cylinder driving scheme, there is conducive to the completion of the six speed manual gearbox test-bed precise automatic shift.

\section{Experimental requirements}

As shown in Fig.1 is a typical product running safety life prediction test rig and automatic shift system, Through the gear shift device of the experimental platform, the test products can be controlled to run at different speeds, then the prediction of the life of the parts is carried out, thereby improving the accuracy of the experimental bench and increasing the prediction range of the experimental table. The shift device has six files, respectively, for one, two, three, four, five, reverse, in the test process, gear from one file start variable to five file to achieve different speed, and through finite cycles to measure the life of mechanical parts.

The current most domestic experimental benches still use manual shift, which requires in the process must be man-made real-time observation of the experimental process. In order to liberate people from the machine work, this system can be real-time control by means of computer, so as to 
achieve one, two, three, four, fifth gear and reverse gear continuous repetition of transform In the experimental process, and realize the control of different time and different gear, which is very significant for the car manual gearbox to be automatic shift.

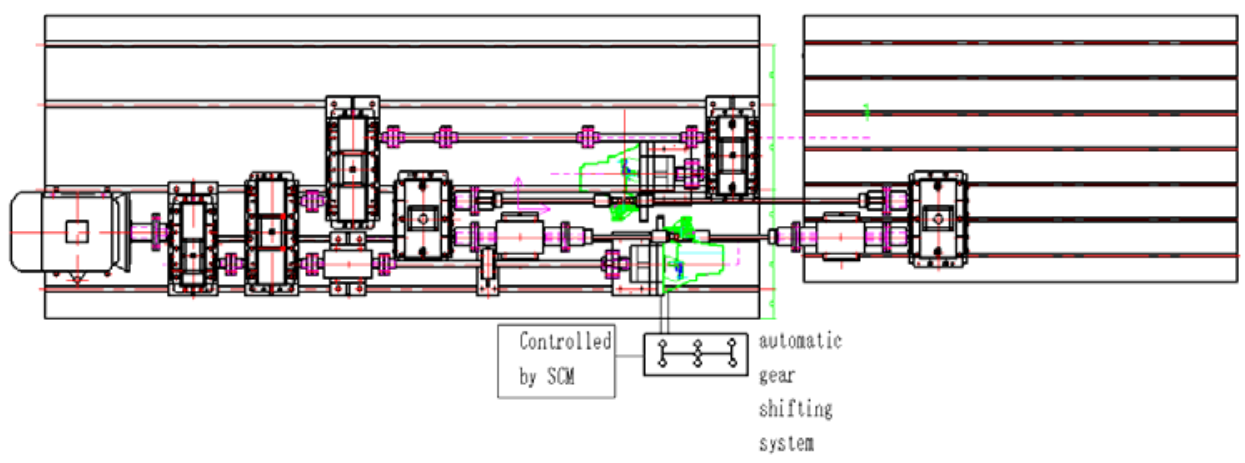

Fig.1 typical product operation safety life prediction technology research

\section{The manual transmission shift movement logic of the test bench}

The manual gearbox control rod's movement from one to five file and reverse file to complete the conversion between the different speeds. Shown in Fig.2 for logic control diagram, it sets control rod before and after the direction of movement for the $\mathrm{Y}$ direction, left and right direction for the X direction. Below are the same.

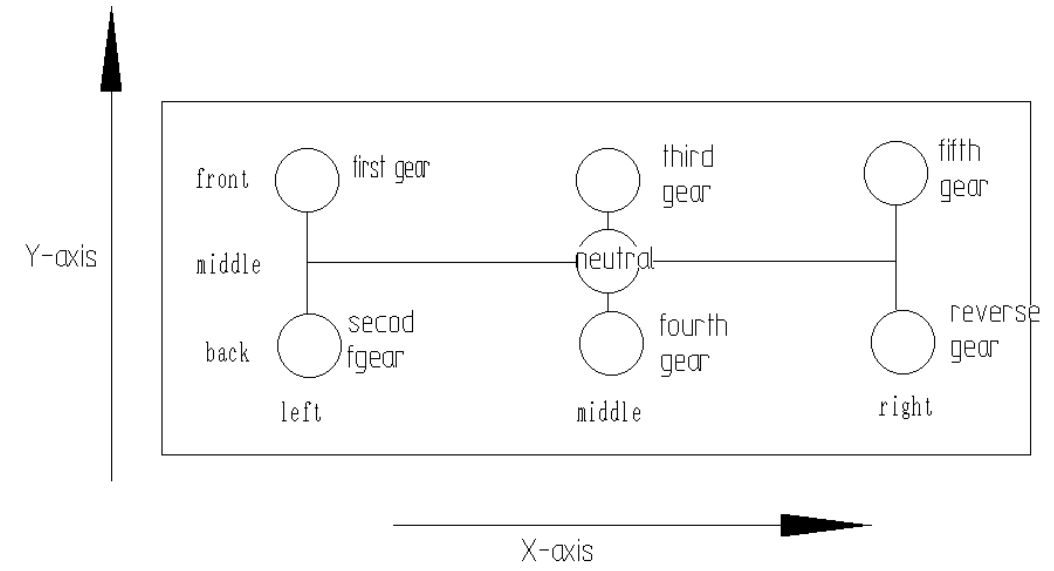

Fig.2 Logic control diagram

The establishment of coordinate system as shown in the Table 1:

Table 1

\begin{tabular}{cccc}
\hline Shifting position & One file & Two file & Three file \\
\hline position & left, front & left, back & middle, former \\
Shifting position & Four file & Five file & Six file \\
position & middle, back & right, front & right, back \\
\hline
\end{tabular}

Set control tap in neutral (middle, middle) position, in order to achieve the shift to one file. Its control movement logic is as follows: first of all, control the platform for motor screw rod, the tap moves from the neutral position (middle, middle) position to the left position (left, middle), and then control the cylinder of the other platform to move the tap to one file position ,thus shifting operation will complete the manipulation of tap. Similarly, the motor screw in board 2 (Fig3) and cylinder in board 3 (Fig3) cooperate with each other, it can be achieved to shift the control tap in any files. Finally, it can be controlled for the motor and cylinder by Single chip, and will be in accordance with the timing automatic shift to achieve the functional requirements of gearshift device. 


\section{Brief introduction of automatic gear shifting system}

\section{Analysis of the principle}

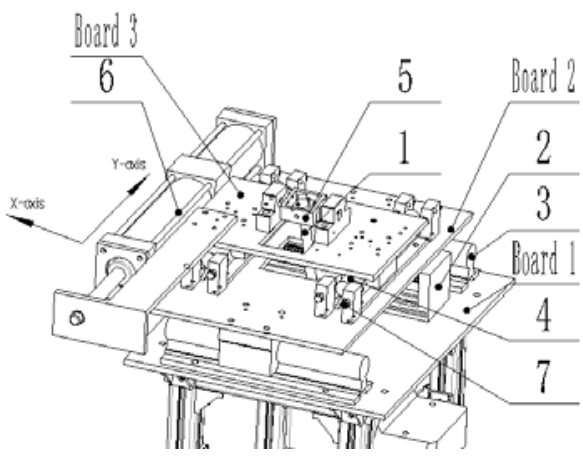

Fog. 3 three-dimensional structure of the principle

1.shifting rod 2.screw 3.4.lead rail 5.top rotary sliding pair 6.three-position cylinder 7.buffer

As shown in the fig.3, the system mainly contains two layers, assuming that the layer between board 1 and board 2 is subsurface, the layer between board 2 and board 3 is upper surface. Place the screw and lead rails on the board 1,when the screw moves, board 1 and board 2 moves with it, as the same time, the top rotary sliding pair begins to turn around and slither, then the shifting rod moves in the direction of X-axis. Place cylinder and lead rails on the board 2, the cylinder make board 2 move, as the same time, the top rotary sliding pair begins to turn around and slither, then the shifting rod moves in the direction of Y-axis. Subsurface fixed on the upper surface.

Two layers are in charge of two different directions severally, namely the shifting rod 's position of front, middle, back and left, middle ,right. Because of screw's high precision and accurate positioning, we choose it to motive the board 2.Before the experiment, distances among the three positions must be calculated, then according to the calculation, the screw rotation can achieve positioning three points. The upper surface used the cylinder to achieve the three points in the direction of Y-axis. The direction of the movement precision is not high, but this direction takes long path, the cylinder can quickly give the long path. A mechanism is also needed to limit the shifting rod, the rotating mechanism is designed based on the cardan joint, and it can make the shifting rod to achieve circular motion.

\section{Data analysis and stroke length'determination of the screw and cylinder}

According to the analysis of the mechanical structure, the shifting rod's length is processed for 230 millimetres long. The top point of the shifting rod can move 190 millimetres long in the direction of $\mathrm{X}$-axis and 480 millimetres long in the direction of Y-axis by measuring. The height of the board 1 (such as in Figure 3) is designed for 97 millimetres long and board 2 (such as in Figure 3) for 480 millimetres long based on the lead rail's height.

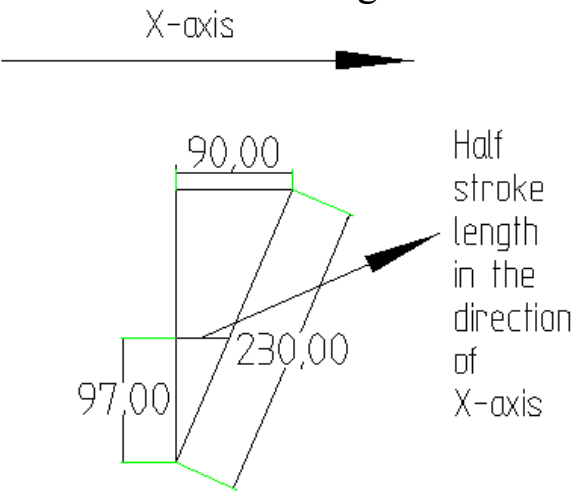

Fig.4 Half stroke length in the direction of X-axis

As the Fig 4 says, figure out the half path in the direction of X-axis by the measuring datas. If the three sides of two tringles is proportional, then the two triangles are similar [7]. According to the theory of the similar tringle:

Half path in the direction of X-axis/95=97/230 
Half path in the direction of $\mathrm{X}=40$

So screw's stroke length $>=2 * 40 \mathrm{~mm}=80 \mathrm{~mm}$

According to the national standards, we choose the stroke length for 100 millimeters long.

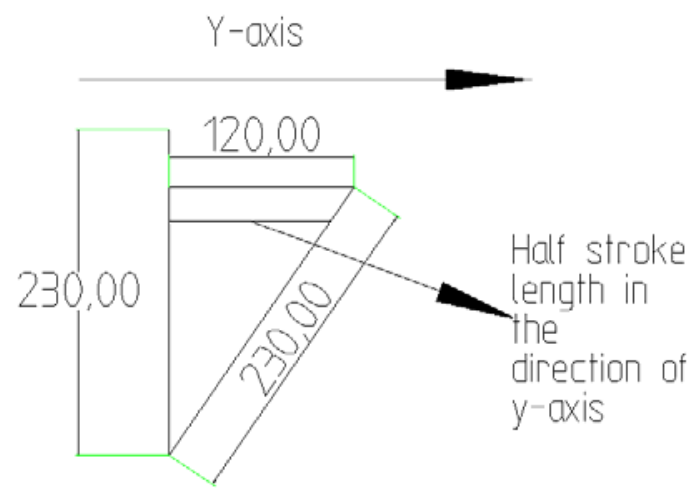

Fig.5 Half stroke length in the direction of Y-axis

As same as the $\mathrm{X}$ direction:

Half path in the direction of $Y$-axis $/ 120 \mathrm{~mm}=165 \mathrm{~mm} / 230 \mathrm{~mm}$

Half path in the direction of $Y=86 \mathrm{~mm}$

So screw's stroke length $>=2 * 86 \mathrm{~mm}=172 \mathrm{~mm}$

According to the national standards, we choose the stroke length for 200 millimeters long.

\section{Mechanical structure}

\section{Mechanical structure in the $\mathrm{X}$ direction}

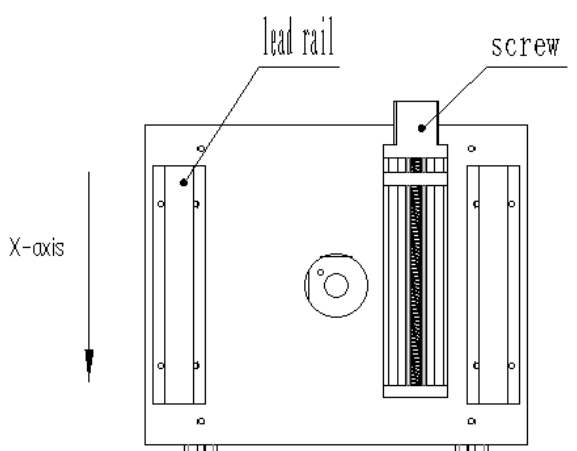

Fig. 6 plane pictures in the $\mathrm{X}$ direction

According to the Fig 6, this layer consists of one screw and one pair of lead rail. This layer's function is to motivate the shifting rod to achieve positing three accurate points. The shifting rod's initial status is empty gear, that means the sliding table of the screw is in the middle position. When shifting the gear, stepper motor can accurately control the sliding table to forward or backward for 40 milimeters long, The balls in the screw guarantee accuracy of the three positions. The lead rails on the two sides are parallel to the screw, so the sliding table can prop up the whole broad to guarantee the system's stability. 


\section{Mechanical structure in the $\mathrm{Y}$ direction}

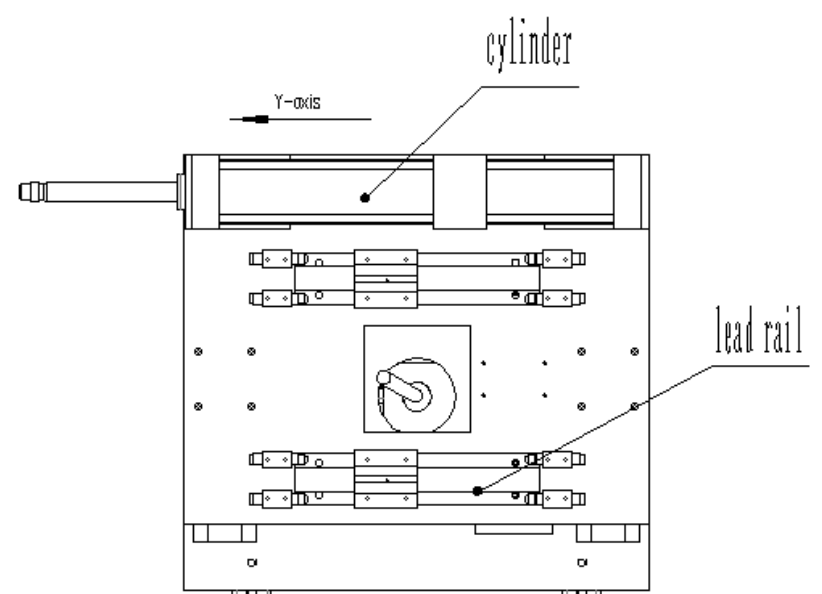

Fig. 7 plane pictures in the $\mathrm{Y}$ direction

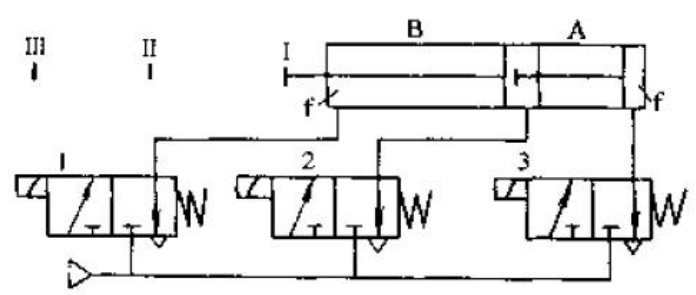

Fig.8 Schematic diagram of three-position cylinder

As shown in the Fig.7, this layer consists of one three-position cylinder and a pair of lead rails. This layer's function is to motivate the shifting rod to achieve positing three points quickly. The schematic diagram of three-position cylinder is shown in the Fig.8, when valve 1 galvanizes, the two psiton rods are all in returned shape, as this time the shifting rod is in the rear position(shown in Fig.2); when valve 3 galvanizes, the piston in cylinder A pushes the piston in cylinder B from the position I to II ; When valve 2 galvanizes, the piston in cylinder B continues to move from II to III, as this time the shifting rod is in front of the poison(shown in Fig.2). When both valve 2 and valve 3 galvanize, the piston in B cylinder is limited to the II position, as this time, the shifting rod is in the middle position(shown in Fig.2)[8].

\section{The design of the top rotating sliding pair}

\section{The necessity of the top rotating sliding pair's design}

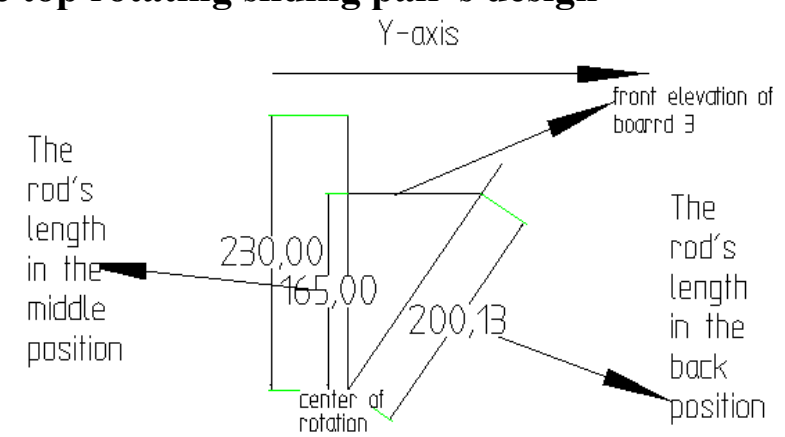

Fig.9 Diagrammatic sketch of the shifting rod's movement

As shown in the Fig.9,take the Y-axis' movement for an example, when the shifting rod is moving, board 3 will move to different gears, corresponding to the length from the rotating point to the board of the shifting rod is different. It's 165 milimeters long in the middle position and 186 
milimeters long in the back position. So it easily brings about interference. In order to solve this problem, we must design a pair of element.

\section{Mechanical structure of the top rotating sliding pair}

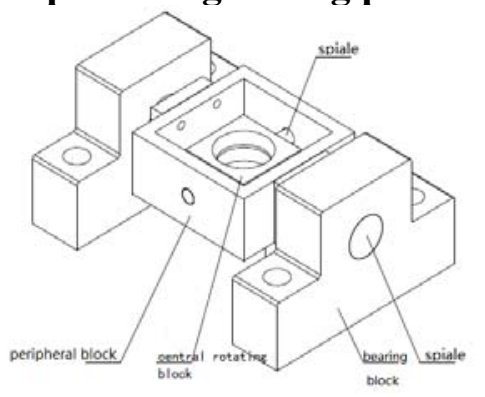

Fig. 10 rotating sliding pair

As shown in the Fig.10.The structure in the middle of the pair is central rotating block which is in charge of the X-axis' movement, the top of the shifting rod is sheathed on the central rotating block., then the central roating block is connected to the peripheral block which is in charge of the Y-axis' movement, at last, the peripheral block is connected to the bearing blocks on the both sides. The cylindroid hole in the middle of the rotating block is bevelled in order to reduce the friction force, it's good for the shifting rod's movement, meanwhile, the shifting rod can also be limited.

\section{Control method}

\section{Control method}

By controlling the step motor moving, the shifting rod can move in the direction of X-axis. We use the multiposition cylinder to make the shifting rod move in the direction of Y-axis. Then we can achieve the aim of the automatic gear switch by all above. The LCD screen of the control panel can show us every realtime parameter of the equipment, it consists of the time of each gear, the cycle index, the main moving time, the shift speed, etc. We can change operation parameters (such as the shift speed and the cycle index, etc) by setting the buttons.

\section{Hardware selection}

Arduino is a popular electronic interactive platform.it is based on the MCU system, and easy to use. It has the advantage of functional diversity, the low price and so on.it is widely used in the electronic system design and the interactive product development [9].

We choose the SCM Arduino uno (simple I/O platform based on the open source code), the control panel (including the LCD display and parameter settings), a feedback device (the position stroke switch), the TT-NRM and one stepper motor driver according to the control requirements.

\section{Control implementation}

SCM control stepper motor drive and then to control the stepper motor to adjust the shifting rod's stroke in X-axis direction, it also controls TT-NRM and then to control electromagnetic valve so that three-position cylinder can adjust the shifting rod's stroke in Y-axis direction according to the requirements, the feedback switch is installed in the three-position's way, so we can judge the piston rod's through the feedback switch. Compared the stroke required to the position feedback, single chip microcomputer continues to control the cylinder to move, it can't stop until the cylinder stroke position and the expected shift required positions agree cylinder stop action. The flowsheet of automatic gear shifting is shown as Fig.11. 


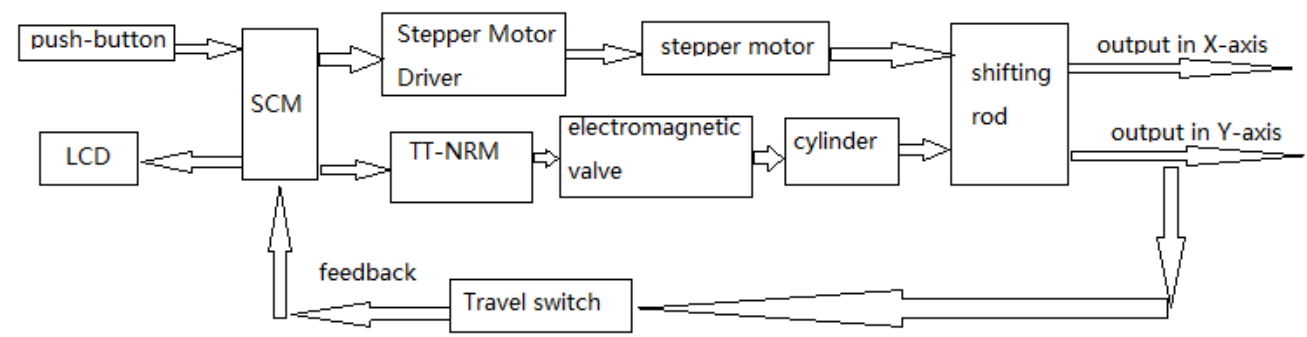

Fig.11 The flowsheet of automatic gear shifting

\section{Experiment}

\section{Empirical method}

Experiments was conducted in two different conditions, once for the system alone without a load independent operation, another was tested combined with experiment table. Stipulate the runtime in different gears in advance, plan the movement logic and write program according to it. And then observe the difference between the expected and reality.

\section{Experimental result}

Through the observation of the experimental phenomena. The results show that shifting rod can stay any gear for a required time, the system can shift the gear quickly and accurately. To a certain extent, the automatic shifting can improve the shift quality instead of manpower for shifting circularly and regularly.

\section{Conclusion}

In the manual shift of manual transmission test bench, this paper puts forward automatic shift system platform. Adopting double layer and biaxial design, driving by screw and cylinder, at last the stepper motor and the solenoid valve controlled by Arduino uno. The results prove that the Institution can controls the joystick accurately and make the transmission shift periodically, circularly and efficiently.

\section{References}

[1] Wang Minghong,Chen Hongxiang.A control system design of automobile manual transmission[J].Proceedings of national mechanical design in the sixteenth year.2012:1-2

[2] Yin Minggui.10 Speed Transmission Control System Design[J].Mechanical Engineering \& Automation.2012(4):1-4

[3] Chen Jingwei,Song Kehui.Design and optimization of automobile manual transmission[J]. Science and Technology Consulting Herald.2011(30):5-8

[4] Ge Anlin,Study on AMT Gear Shifting Behavior [J].AUTOMOBILE TECHNOLOGY

[5] Cheng Qin. Analysis of automobile design manual gearbox gearshift test research and shift quality[J].Dissertation.2009:2-4

[6] Lu Yongsheng. Application of AMT automatic transmission on the bus in the city[J].BUS TECHNOLOGY AND RESEARCH.2006(01):3-4

[7] Lu Xianfu.On exploring of teaching[J].JOURNAL OF ANSHUN TEACHERS COLLEGE. 2005,7(4):3-6

[8] Information on http://wenku.baidu.com/link

[9] Cai Ruiyan.Principle and application of Arduino[J].Electronic Design Engineering.2012, 20(16) 